\title{
COMMENT ON "WAVE PROPAGATION EFFECTS AND THE EARTH'S STRUCTURE IN THE LOWER MANTLE"
}

Christopher J. Young and Thorne Lay

Department of Geological Sciences, University of Michigan

Abstract. In a recent contribution, Haddon and Buchbinder introduced a new shear velocity model for the lower mantle which, combined with effects of velocity inhomogeneity at the base of the mantle, they concluded to be consistent with observed SH arrivals preceding the core reflected phase ScSH at distances beyond $70^{\circ}$. Using Kirchhoff wave theory, they computed synthetic waveforms for the radially symmetric version of their model (which has a $1.4 \%$ discontinuous velocity decrease at $2300 \mathrm{~km}$ depth) that seem to match some SH data, even without allowing for lateral heterogeneity. However, synthetic waveforms for this model computed using generalized ray theory and reflectivity do not match the Kirchhoff synthetics or the data, casting doubt upon the conclusions drawn in their study. Earlier models by Lay and Helmberger, involving a $2.75 \%$ discontinuous velocity increase at a depth of about $2600 \mathrm{~km}$, require only slight modification in order to match all of the SH wave observations.

\section{Introduction}

Based on detailed synthetic waveform modeling of observations of a separate arrival between SH and ScSH in the distance range $70-82^{\circ}$ and of $\mathrm{SH}$ waveform distortions in the range $89-92^{\circ}$, Lay and Helmberger [1983] proposed the existence of a $2.75 \%$ abrupt shear velocity increase about $280 \mathrm{~km}$ above the core-mantle boundary. A subsequent study [Young and Lay, 1987] has added to the S wave data set and strengthened the hypothesis that such a velocity increase occurs on a global scale. Haddon and Buchbinder [1986], noting variations in amplitude of the arrival intermediate to $\mathrm{SH}$ and $\mathrm{ScSH}$ for distances less than $78^{\circ}$, have explored alternate lower mantle velocity models using synthetic waveform calculations to determine whether the intermediate arrival can instead be interpreted as an effect of wave propagation in the inhomogeneous layer, designated $D^{\prime \prime}$, at the base of the mantle. The evidence for significant heterogeneity in $D^{n}$ is strong [Sacks et al., 1979; Sengupta et al., 1981; Haddon, 1982; Clayton and Comer, 1983; Dziewonski, 1984] and it is reasonable to assume that the heterogeneity will introduce scatter into the amplitudes and travel times of phases traversing $D^{n}$. However, in order to match the SH wave observations, which have an extra discrete arrival, Haddon and Buchbinder [1986] still require a discontinuous radial structure to provide this extra arrival which is then augmented or diminished by focusing and scattering from lateral heterogeneity within $D^{n}$. Their basic radial model, Dl (Figure 1), corresponds to accepted radially symmetric reference models (e.g. JB) down to $2300 \mathrm{~km}$ where it has an abrupt $1.4 \%$ decrease in velocity, followed by a linear velocity increase which extends to the coremantle boundary. Because the averaged velocity structure of model D1 is not equivalent to any accepted radial reference Earth model, D1 does not accurately predict the travel times for lower mantle phases such as ScS. Nevertheless, the

Copyright 1987 by the American Geophysical Union.

Paper number 7L7132.

$0094-8276 / 87 / 007 \mathrm{~L}-7132 \$ 03.00$ synthetic waveforms computed by Haddon and Buchbinder for model D1 do match some of the SH wave observations of Lay and Helmberger [1983], even without considering the effects of $D^{n}$ heterogeneity. The purpose of this comment is to demonstrate that the synthetic waveforms presented by Haddon and Buchbinder for the radially symmetric version of model D1 seem to be inaccurate, and to explore the nature of alternative lower mantle models that more closely match the data.

\section{Discussion}

Figure 1 shows the deep mantle $S$ velocity models D1 [Haddon and Buchbinder, 1986] and SLHO [Lay and Helmberger, 1983] along with SH travel time curves calculated for each. The travel time curve for SLHO shows a triplication due to the discontinuous velocity increase at $2600 \mathrm{~km}$. The cd branch of this triplication causes an arrival intermediate to Sab and ScS in the distance range 70$82^{\circ}$ and it is this arrival which Haddon and Buchbinder seek to match with their model DI.

Because model D1 has a discontinuous decrease in velocity, the corresponding travel time curve has a shadow zone for the direct $S$ arrival extending from $80-88^{\circ}$. This shadow zone is illuminated by diffraction from the discontinuity at $2300 \mathrm{~km}$. In the distance range less than $80^{\circ}$, a reflected branch from the discontinuity can be seen. The separation between this branch and the $S$ branch decreases from a few seconds near $70^{\circ}$ to 0 seconds at $80^{\circ}$ where the branches merge. It is apparent from the timing of this reflected branch that it cannot account for the arrivals attributed to the cd branch of the triplication shown in the travel time curve for model SLHO. The travel time curve for Dl shown in Figure 3 of Haddon and Buchbinder [1986] does not appear to give correct travel times for this reflected branch.

In order to more clearly demonstrate the effects of the two models on deep mantle phases, an intermediate step in the calculation of generalized ray theory synthetic seismograms [Helmberger, 1974] is shown here. Figure 2 shows profiles of the SH displacement step responses for models DI and SLHO. These step responses represent the Earth structure transfer function, $M(t)$ in the convolution equation for the computation of a synthetic seismogram [Helmberger and Burdick, 1979]:

$$
S S(t)=S(t) * M(t) * E(t)
$$

where $S S(t)$ is the synthetic seismogram, $S(t)$ is the convolution of the receiver and attenuation terms, and $E(t)$ is the source term (in this case a step function).

The step responses in the distance range $72-84^{\circ}$ for the SLHO profile show three sub-steps due to the Sab, Scd (indicated by arrows), and ScS arrivals. The arrivals move through and interfere with each other in the range $84-88^{\circ}$ and beyond $88^{\circ}$ there are only the two arrivals, Scd, which has crossed in front, and Sab. The large amplitude of the $\mathrm{Sab}$ arrival beyond the crossover distance in these steps leads to a large secondary arrival in the synthetic seismograms which Schlittenhardt et al. [1985] have shown is not 

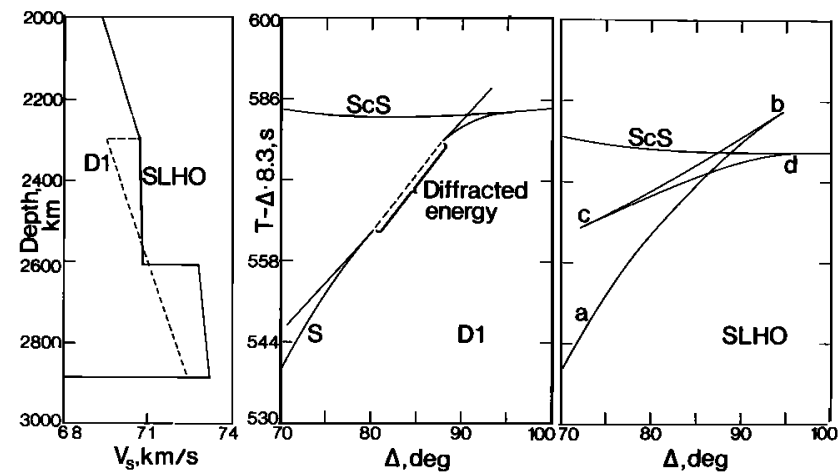

Fig. 1. (left) Depth vs. shear velocity profiles for the models D1 and SLHO. (right) Travel time curves for the models calculated by ray tracing with a source depth of $\mathbf{5 8 0}$ km.

observed in some diffracted $S$ wave data, but this problem seems to have been resolved by a slight modification of model SLHO [Lay, 1985; Young and Lay, 1987], which we will discuss later.

In the D1 profile, the sharp arrival of the geometric $S$ ray can clearly be seen on all of the step responses out to about $80^{\circ}$, where the shadow zone begins. There is no sharp arrival in the shadow zone due to the lack of a geometric ray, but there is an emergent diffracted arrival. A sharp arrival can again be seen for distances beyond $88^{\circ}$, which is past the shadow zone. The ScS arrival (trailing $S$ by about 40 seconds at $72^{\circ}$ ) can also be clearly seen. Intermediate to these two features is a smaller arrival (indicated by arrows) with negative polarity which is the wide angle reflection from the discontinuous velocity decrease at $2300 \mathrm{~km}$ depth. There are two significant problems with attempting to use this arrival as an explanation for the intermediate arrivals shown in the Lay and Helmberger [1983] data set. First, as mentioned above, the timing is wrong: this branch is much too close to $S$ at the necessary distance range because the discontinuity is so far $(600 \mathrm{~km})$ above the core. This implies that for a long period instrument response, this step will not even show up on the synthetic seismogram. Second, even if an arrival were seen, the polarity would be wrong.

Figure 3 shows profiles of final SH synthetic seismograms for D1 and SLHO calculated using reflectivity theory, which should adequately account for diffracted effects. The D1 synthetics should agree with those shown in the center profile of Figure 4 of Haddon and Buchbinder [1986] but do not. The Haddon and Buchbinder profile, calculated using Kirchhoff theory, shows an arrival intermediate to $S$ and $\mathrm{ScS}$ that they attributed to diffraction from the discontinuity. Neither our reflectivity synthetics nor our generalized ray theory synthetics show any such arrival. The fact that our synthetics show no intermediate arrival suggests that diffraction effects are not significant in this profile other than for producing arrivals in the shadow zone, and this idea is supported by the close agreement of reflectivity synthetics with generalized ray theory synthetics for D1.

An intermediate arrival is clearly visible in the SLHO

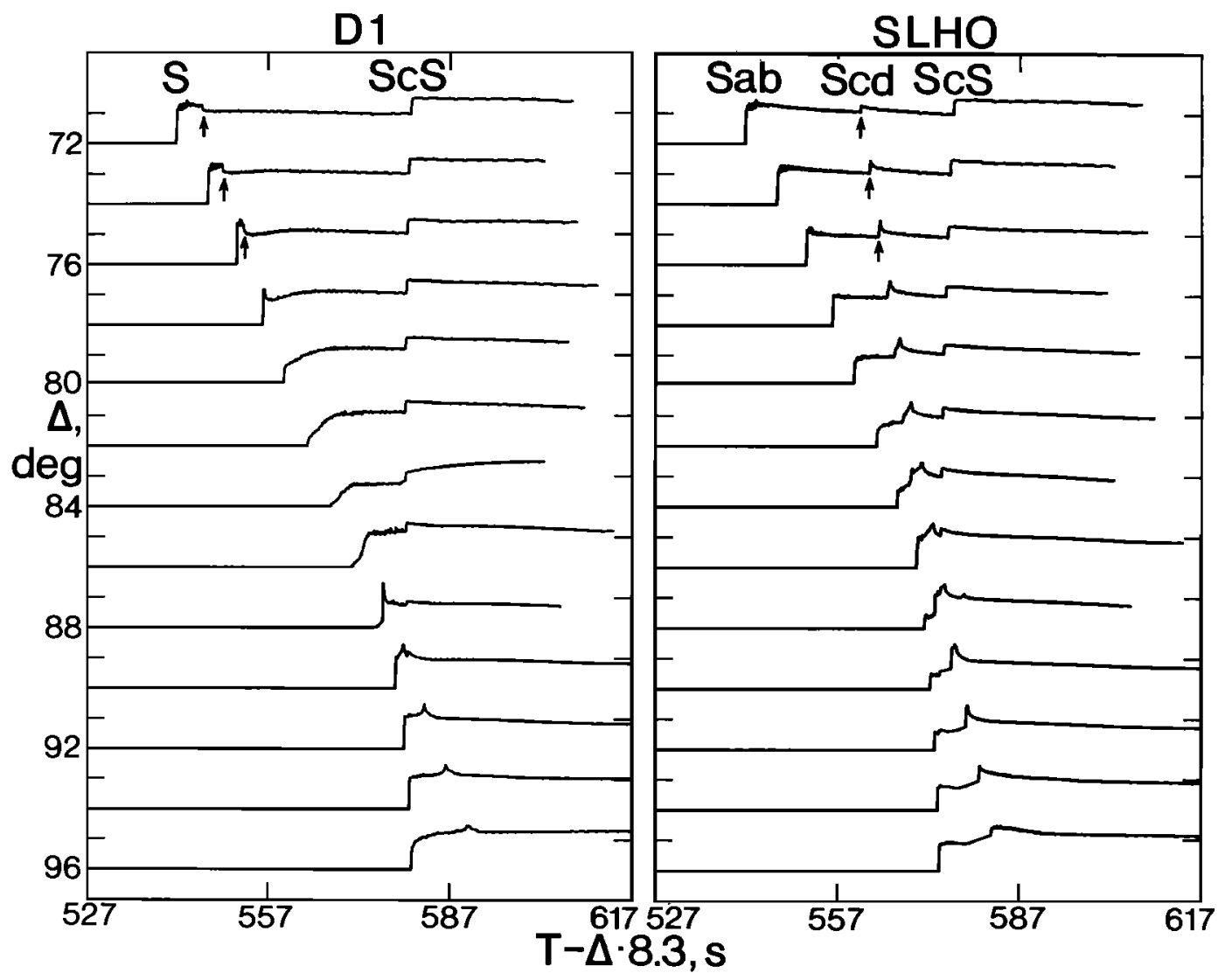

Fig. 2. Profiles of displacement step responses for the DI and SLHO profiles. Intermediate arrivals are indicated by arrows. Steps were calculated using generalized ray theory with a source depth of $600 \mathrm{~km}$. 

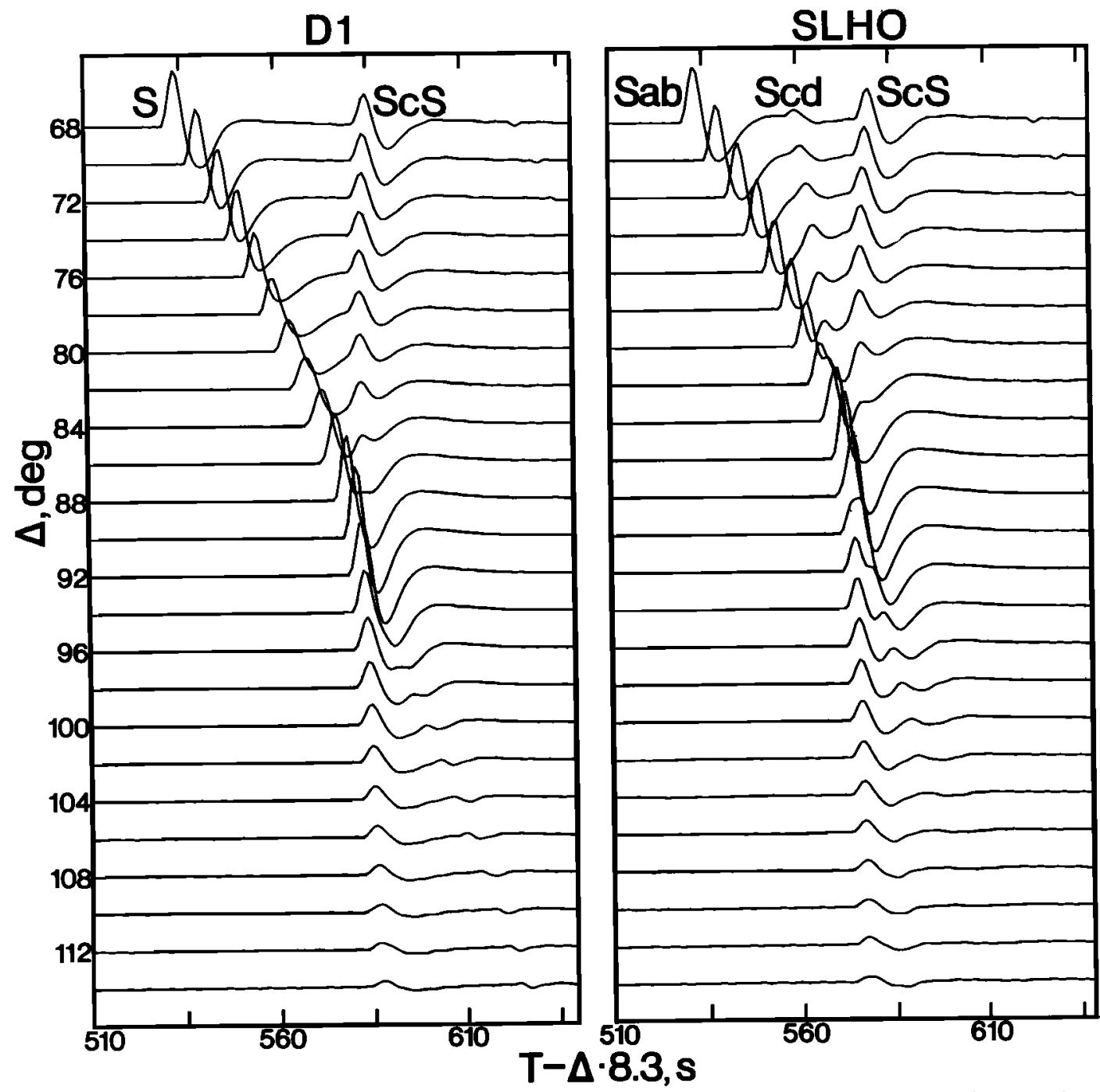

Fig. 3. Profiles of synthetics long period SH seismograms for the Dl and SLHO profiles. These synthetics were calculated using reflectivity theory (with a source depth of $580 \mathrm{~km}$ ) and so should include diffraction effects.

profile, as are the interference effects caused by the crossing over of the ab and cd branches of the triplication. Beyond $92^{\circ}$, the ab branch is manifested as a fairly strong secondary arrival which, as mentioned above, is not observed in some data. Modifying the shear velocity discontinuity model by changing the gradient beneath the discontinuity from slightly positive to slightly negative yields synthetics which provide a good fit to data at diffracted distances [Lay, 1985; Young and Lay, 1987]. The new model, SYL, is shown in Figure 4 along with a profile of SH reflectivity synthetics. The synthetics are nearly identical to those for model SLHO for the distance range $68-88^{\circ}$, the range over which SLHO fits the data well, but show important differences beyond $88^{\circ}$. For the new model, in the distance range greater than $88^{\circ}$ the Sab-Scd time is smaller, as is the Sab/Scd amplitude ratio and this leads to a better fit to the data. These effects are directly due to the slight velocity gradient modifications. The negative gradient in $D^{n}$ causes more energy to turn below the discontinuity leading to a decrease in the Sab/Scd amplitude ratio. The reduced gradient also slows down the Scd energy, while the $\mathrm{Sab}$ energy arrives earlier due to the increased velocity gradient above the discontinuity, and both of these effects decrease the Scd-Sab differential time.

The SLHO model of Lay and Helmberger [1983] was proposed to fit a data set of SH seismograms spanning the distance range from about $70-92^{\circ}$. Due to the nature of that study, which relied upon synthetic waveform modeling to determine deep Earth structure, the size and depth of the shear velocity discontinuity $(2.75 \pm 0.25 \%, 2608 \pm 20 \mathrm{~km})$ were constrained fairly well but the velocity gradients above and below the discontinuity were not, nor was the sharpness of the discontinuity. The gradient within $D^{n}$ was modeled as slightly positive to agree with long-period amplitude ratios of ScSH/SH amplitudes but this is not a very tight constraint, and it is also satisfied by the SYL model. The data at diffracted distances (beyond $95^{\circ}$ ), provide better constraints on this gradient, requiring the negative gradient in SYL. The gradient above the discontinuity is poorly constrained. The choice of a reduction in the lower mantle velocity gradient beginning at $2300 \mathrm{~km}$ and extending to the discontinuity at $2620 \mathrm{~km}$ in model SYL represents one simple way to keep the average ScS travel times consistent with the JB model; certainly there are many other ways to 

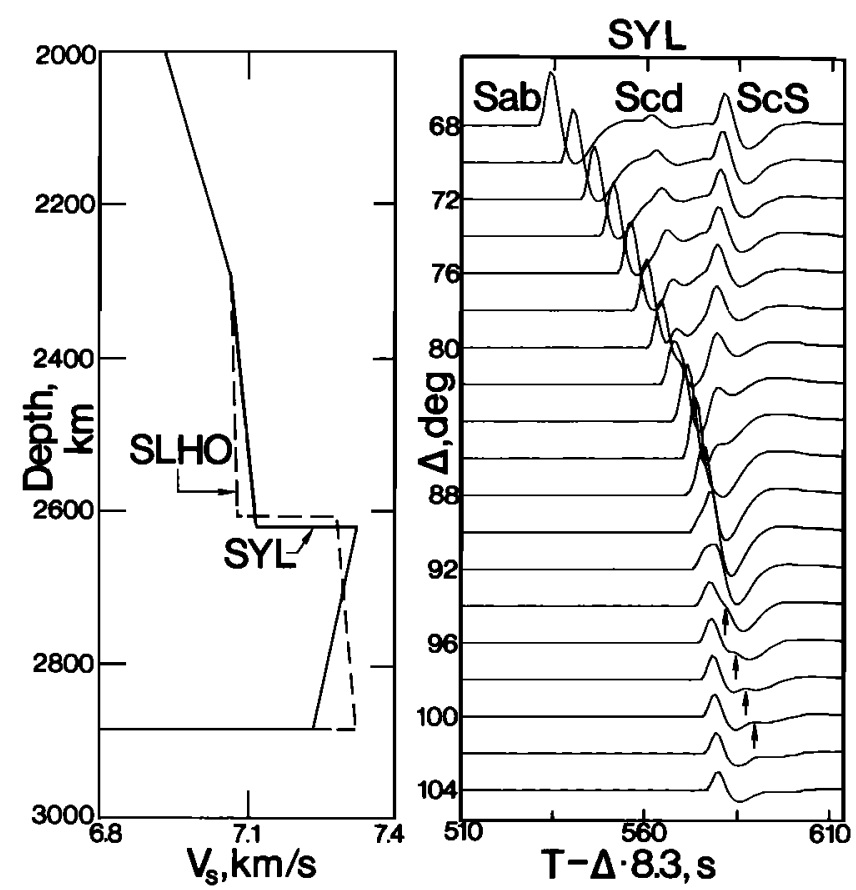

Fig. 4. (left) Depth vs. shear velocity profiles of lower mantle shear velocity discontinuity models SLHO and SYL. (right) Profile of synthetic long period SH seismograms for the SYL model. Arrows indicate the diminished secondary diffracted arrival. The synthetics were calculated using reflectivity theory with a source depth of $580 \mathrm{~km}$.

do this. We do not feel that the precise velocity gradient above the discontinuity is particularly diagnostic of the $D^{n}$ discontinuity models because it is poorly constrained by our method of modeling. Lateral heterogeneity in $D^{n}$ probably does account for amplitude and travel time variations of the $S$ wave arrivals, but it appears that if deep mantle structure is responsible for this complexity, a concentrated velocity increase is required to match the data. Alternate possible explanations of these data as the result of diffraction by slabs penetrating into the lower mantle have yet to be fully explored.

\section{Conclusions}

The proposed model, D1, of Haddon and Buchbinder [1986] does not seem to provide a viable explanation for SH data at distances beyond $70^{\circ}$. Even allowing for lateral heterogeneity, which we would agree affects the data set, a different sort of model is required to account for the additional arrivals and waveform distortions seen. The SH wave observations [Lay and Helmberger, 1983; Lay, 1986; Young and Lay, 1987] have yet to be adequately explained by structures that do not have deep mantle abrupt shear velocity increases. With a slightly revised model, SYL, it is possible to fit SH data beyond $95^{\circ}$ which could not be fit by model SLHO. The size and depth of the shear velocity increase are fairly well constrained, the abruptness of the increase and the velocity gradient beneath it are less so, and the velocity gradient above it is poorly constrained.

Acknowledgements. Helpful comments for improving the manuscript were provided by Lisa Donaghe and Susan Schwartz. We thank R. A. W. Haddon for providing us with Kirchhoff synthetics for the Dl model. C. J. Young was supported by an NSF Graduate Fellowship. This research was supported by the Alfred P. Sloan Foundation, a Shell Faculty Career Initiation Grant, and NSF Grant EAR8451715.

\section{References}

Clayton, R. W., and R. P. Comer, A tomographic analysis of mantle heterogeneities from body wave travel time data (abstract), Eos, Trans., AGU, 64, 776, 1983.

Dziewonski, A. M., Mapping the lower mantle: determination of lateral heterogeneity in $\mathbf{P}$ velocity up to degree and order 6, J. Geophys. Res., 89, 5929-5952, 1984.

Haddon, R. A. W., Evidence for inhomogeneities near the core-mantle boundary, Phil. Trans. Roy. Soc. Lond. A 306, 61-70, 1982.

Haddon, R. A. W., and G. G. R. Buchbinder, Wave propagation effects and the Earth's structure in the lower mantle, Geophys. Res. Lett. 13, 1489-1492, 1986.

Helmberger, D. V., Generalized ray theory for shear dislocations, Bull. Seismol. Soc. Am., 64, 45-64, 1974.

Helmberger, D. V., and L. J. Burdick, Synthetic Seismograms, Ann Rev. Earth Planet. Sci. 7, 417-442, 1979.

Lay, $T$., Analysis of diffracted $S$ waves traversing a region with a lower mantle shear velocity discontinuity (abstract), Eos, Trans., ACU, 66, 310, 1985.

Lay, T., Evidence for a lower mantle shear velocity discontinuity in S and sS phases, Geophys. Res. Lett., 13, 1493-1496, 1986.

Lay, T., and D. V. Helmberger, A lower mantle S-wave triplication and the shear velocity structure of $D^{n}$, Geophys. J.R. Astron. Soc., 75, 799-838, 1983.

Sacks, I. S., J. A. Snoke, and L. Beach, Lateral heterogeneity at the base of the mantle revealed by observations of amplitudes of PKP phases, Geophys. J. R. Astron. Soc., 59, 379-387, 1979.

Schlittenhardt, J., J. Schweitzer, and G. Muller, Evidence against a discontinuity at the top of $\mathrm{D}^{n}$, Geophys. J.R. Astron. Soc., 81, 295-306, 1985.

Sengupta, M. K., R. E. Hassell, and R. W. Ward, Threedimensional seismic velocity structure of the Earth's mantle using body wave travel times from intra-plate and deep-focus earthquakes, J. Geophys. Res., 86, 3913-3934, 1981.

Young, C. J., and T. Lay, Evidence for a shear velocity discontinuity in the lower mantle beneath India and the Indian Ocean, Phys. Earth Planet. Inter., in press, 1986.

Thorne Lay and C. J. Young, Seismological Laboratory, Department of Geological Sciences, 1006 C. C. Little Bldg., University of Michigan, Ann Arbor, MI 48109

(Received February 4, 1987; accepted February 14, 1987.) 